\title{
Case Studies of Patients Successfully and Unsuccessfully Managed Pre- and Post-Fontan Procedure
}

\author{
Yasuo ONO, ${ }^{1} \mathrm{MD}$, and Norie Mitsushita, ${ }^{1} \mathrm{MD}$
}

\begin{abstract}
SUMMARY
As a result of advances and modifications in surgical procedures and the development of drugs for pulmonary arterial hypertension, many patients who have undergone Fontan procedures are able to enjoy good quality of life, without pulmonary arterial hypertension and severe complications. In Shizuoka Children's Hospital, drugs for pulmonary arterial hypertension have long been given to Fontan candidates and patients with established Fontan circulation to maintain sufficient pulmonary blood flow and suppress pulmonary arterial hypertension. We present three typical cases that were treated with anti-pulmonary hypertensive drugs before or after Fontan procedure. The first case had asplenia syndrome, and a single ventricle with major aortopulmonary collateral arteries. Anti-pulmonary hypertensive therapy permitted a Fontan procedure and maintained a good long-term quality of life. The second case was a Down syndrome patient who had progressive cyanosis after a Fontan operation. Anti-pulmonary hypertensive therapy improved cyanosis. The third case suffered from protein-losing enteropathy, for which all procedures and medical therapies were ineffective. Fontan candidates and patients with Fontan circulation have varied anatomical backgrounds and pulmonary properties. We must identify the conditions that lead to successful Fontan procedure and Fontan circulation correction, as well as conditions that result in failed Fontan procedure and poorly-controlled Fontan circulation. (Int Heart J 2015; 56: S31-S34)
\end{abstract}

Key words: Pulmonary hypertension, Cardiac malformation, Fenestration, Beraprost, Bosentan, Sildenafil

$\mathrm{T}$ The Fontan procedure is now performed worldwide to treat several complex congenital heart abnormalities. For both Fontan candidates and patients with Fontan circulation, it is important to maintain good pulmonary blood flow and a good pulmonary vascular bed. In this respect, the recent development of vasodilators for treating pulmonary arterial hypertension provides us with expanded therapeutic choices for managing pulmonary arterial hypertension. However, insufficient data have been collected to determine whether vasodilators positively affect the pulmonary vasculature and a patient's prognosis. Clinically, Shizuoka Children's Hospital has been giving vasodilators to Fontan candidates and patients with Fontan circulation to improve the pulmonary blood flow or function since 2001.

We present the cases of three Fontan candidates. The first case successfully underwent the Fontan procedure and obtained good long-term quality of life. The second case was a patient with Down syndrome for whom a venovenous connection was established and vasodilators were particularly effective for decreasing the venovenous shunt flow and increasing appropriate pulmonary blood flow. The third case was a patient with hypoplastic left heart syndrome and cor triatriatum, as well as protein-losing enteropathy (PLE).

\section{Case Reports}

Case 1: At 7 months of age, the patient presented with asplenia syndrome, single right ventricle, single atrium, common atrioventricular canal defect, pulmonary atresia, major aortopulmonary collateral arteries (MAPCAs), and partial anomalous pulmonary venous connection (PAPVC). We made the diagnosis and considered the patient's cavopulmonary and systemic circulation difficult to control. Angiography at that time revealed an oxygen saturation of $76 \%$ and a total of six MAPCAs (three vessels in the right lung and three vessels in the left lung) (Figure 1A).

Two months later, unifocalization for MAPCA was performed, and a right modified Blalock-Taussig shunt $(5 \mathrm{~mm})$ was established to improve the pulmonary blood flow. Cardiac catheterization performed at the age of 15 months, after unifocalization, revealed high mean pulmonary arterial pressure (23/26/26 mmHg, Qp/Qs = 1.12, and pulmonary vascular resistance $(\mathrm{Rp})=4.9$ Wood $\left.\mathrm{U} \times \mathrm{m}^{2}\right)$, indicating that a Glenn procedure would be very difficult. Instead, to treat the pulmonary hypertension, sildenafil, starting at a dose of $0.1 \mathrm{mg} / \mathrm{kg} / \mathrm{day}$, and home oxygen therapy (HOT) were given, followed by bosentan, starting at a dose of $0.3 \mathrm{mg} / \mathrm{kg} / \mathrm{day}$ (Figure 1B). The patient was followed, and the medication continued, for 10 months. Follow-up cardiac catheterization was performed at 25 months of age. As a result of therapy, the pulmonary arterial

From the ${ }^{1}$ Department of Cardiology, Shizuoka Children's Hospital, Shizuoka, Japan.

Address for correspondence: Yasuo Ono, MD, Department of Cardiology, Shizuoka Children's Hospital, 860 Urushiyama, Aoi-ku, Shizuoka 420-8660 Japan. E-mail: Yasuo-ono@i.shizuoka-pho.jp

Received for publication October 31, 2014. Accepted October 31, 2014

Relesed online on J-STAGE March 19, 2015.

All rights reserved by the International Heart Journal Association. 

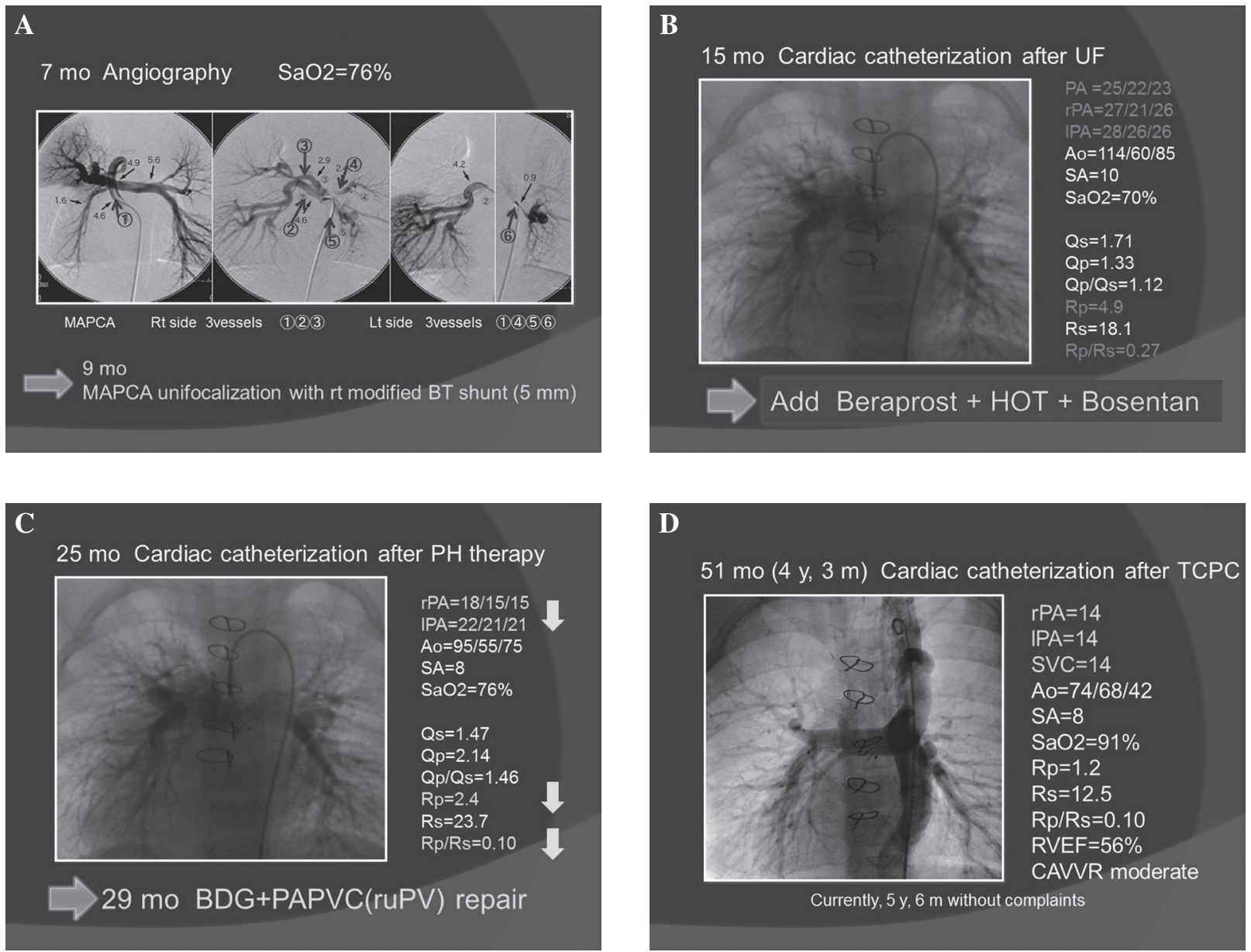

Figure 1. Angiography of Case 1 at 7 months old (A). Angiography and catheter data of case 1 at 15 months old, after unifocalization (B). Angiography and catheter data of case 1 at 25 months old, after pulmonary hypertensive therapy (C). Angiography and catheter data of case 1 at 51 months old, after $\operatorname{TCPC}(\mathbf{D})$

pressure decreased to $15 / 21 \mathrm{mmHg}$, the Rp decreased to 2.4 Wood $\mathrm{U} \times \mathrm{m}^{2}$, and the Rp/systemic resistance (Rs) decreased to 0.10 (Figure 1C). Having determined that the patient could tolerate a Glenn procedure, a bidirectional Glenn procedure and PAPVC repair were done at the age of 29 months. At 33 months, cardiac catheterization was performed. The right and left pulmonary arterial pressures were 10 and $11 \mathrm{mmHg}$, respectively, and the superior vena cava pressure was $11 \mathrm{mmHg}$. The $\mathrm{Rp}$ was 2.1 and the $\mathrm{Rp} / \mathrm{Rs}$ was 0.13 , which indicated that the pulmonary vascular bed was being sufficiently maintained and that the pulmonary blood flow through the Glenn shunt was sufficient. Having confirmed that the patient's pulmonary arterial pressure and central pulmonary venous pressure had decreased sufficiently, the patient was deemed able to tolerate a Fontan procedure, although concerns remained about the condition of the patient's pulmonary vascular bed. At the age of 36 months, a fenestrated total cavopulmonary connection (TCPC) was performed. At 51 months, cardiac catheterization was performed, which revealed right and left pulmonary arterial pressures and superior vena cava pressures of $14 \mathrm{mmHg}$, indicating sufficient control, although moderate common atrioventricular valve regurgitation remained (Figure 1D). At 66 months, the patient had no complaints. Cardiac catheterization revealed that the fenestration had opened, with $91 \%$ oxygen saturation, although mild collaterals developed in the right pulmonary venous vasculature. Closing the fenestration raised the oxygen saturation to $93 \%$. Currently, we are observing his clinical course.

This was a difficult anomaly case, with asplenia, single right ventricle, single atrium, common atrioventricular canal defect, pulmonary atresia, MAPCA, and a PAPVC, but pulmonary hypertension treatment using beraprost, home oxygen therapy, and bosentan decreased his pulmonary hypertension to the point that a Fontan procedure could be performed.

Case 2: The patient had Down's syndrome and tricuspid atresia with pulmonary hypertension. Cardiac catheterization performed at 42 days of age revealed a Qp/Qs of 2.0, pulmonary artery pressure (S/D/M) of 39/22/30, Rp of 4.9 Wood $U \times$ $\mathrm{m}^{2}$, and $\mathrm{Rp} / \mathrm{Rs}$ of 0.39 . Pulmonary arterial banding was performed to treat the elevated pulmonary arterial pressure. The banding rapidly led to stenosis of the right ventricular outflow tract, which caused spells of hypoxia. At the age of 5 months, an emergency cardiac catheterization revealed a mean pulmonary arterial pressure of $15 \mathrm{mmHg}$ and $\mathrm{Rp}=1.0 \mathrm{Wood} \mathrm{U} \times \mathrm{m}^{2}$. To provide smooth pulmonary blood flow, a bidirectional Glenn procedure and an invasive pulmonary arterioplasty were 
performed. Postoperatively, the patient required oxygen and nitric oxide (NO) for an extended period. Without oxygen and $\mathrm{NO}$, the oxygen saturation rate decreased steeply to approximately $50-60 \%$ and we were concerned about severe VV shunts developing. At post-operative day 18, cardiac catheterization was performed, revealing that blood flow through the Glenn shunt was sufficiently maintained and very minor ventriculovenous (VV) shunts had developed, which would have suggested deterioration of the pulmonary vascular bed in large numbers. To prevent the possibility of a deteriorated pulmonary vascular bed, we started beraprost therapy and HOT at 6 months of age. At the age of 16 months, cardiac catheterization was performed, and revealed a low oxygen saturation level of $77 \%$ as well as a low left pulmonary arterial pressure of 9 $\mathrm{mmHg}$, low right pulmonary arterial pressure of $10 \mathrm{mmHg}$, and an Rp of 2.1 Wood $\mathrm{U} \times \mathrm{m}^{2}$. TCPC was performed at 18 months. At 29 months, cardiac catheterization revealed left and right pulmonary arterial pressures of 13 and $14 \mathrm{mmHg}$, respectively, and sufficient oxygen saturation at $93 \%$. Therefore, we stopped HOT and increased the dosage of beraprost. The patient subsequently gained weight. When the patient was 8 years old, oxygen saturation decreased to $88 \%$. An echocardiogram detected abnormal vasculature caused by a venovenous shunt (VVS); therefore, follow-up cardiac catheterization was performed, and the VVS was treated with coil embolization (Figure 2A). This treatment improved the oxygen saturation to $91 \%$.

When we reviewed the oxygen saturation after TCPC, we found that this patient's oxygen saturation level tended to decrease when his clinical condition was not controlled by beraprost. Cardiac catheterization was performed, followed by embolization of the VVS. This increased the oxygen saturation, but only in the short-term. Sildenafil was administered to improve the oxygen saturation level (Figure 2B). As a result, the blood flow increased into the pulmonary vasculature, which led to improved oxygen saturation. Usually, when VVSs become highly developed, it can be difficult to raise the pulmonary arterial pressure without increasing pulmonary blood pressure. However, in this case, beraprost, and subsequently sildenafil, had a positive and continuous impact on the VVSs.

Case 3 (Figure 3): This was a complicated case, with hypo- plastic left heart syndrome and mitral atresia, aortic atresia, and cor triatriatum.

The patient was urgently hospitalized owing to ductal shock. On the following day, a Norwood procedure was performed using a $5 \mathrm{~mm}$ right ventricular-pulmonary artery conduit, while also repairing the cor triatriatum. During the next 4 months, regular echocardiograms suggested decreased pulmonary blood flow through the right ventricular-pulmonary artery (RV-PA) conduit. Cardiac catheterization revealed severe stenosis of this area and massive aortopulmonary collaterals. In addition, the oxygen saturation level had barely changed (Figure $3 \mathrm{~A})$. At the age of 5 months, another RV-PA conduit reconstruction was performed along with bilateral internal thoracic artery ligation, and left fine APCAs cleaning. The patient developed PLE at the age of 6 months, prior to the Glenn and Fontan procedures. Many PLE patients have been shown to have high systemic venous pressure, low cardiac index, high pulmonary vascular resistance, decreased left ventricular systolic function, and elevated systemic ventricular end-diastolic pressure. In addition to the typical symptoms and because many MAPCAs had developed, APCA embolization was performed at 6 and 8 months. Subsequently, the pulmonary arterial pressure was $13 \mathrm{mmHg}$, the oxygen saturation level was as high as $90 \%$, and the Rp/Rs was 0.08 , indicating that the patient was ready for a bidirectional Glenn procedure and pulmonary arterioplasty, which we performed at 8 months. Minor APCA embolizations were performed again at 16 and 18 months. Many MAPCAs had appeared as well; therefore, embolization was performed. However, it is doubtful that this patient's condition was affected by hypoxia, and instead, growing MAPCAs may have been the cause of the disease. Cardiac catheterization performed at 26 months revealed superior vena cava and left and right pulmonary arterial pressures of 13,12 , and $12 \mathrm{mmHg}$, respectively; an oxygen saturation of 87\%; Qp/ Qs of 0.96 ; and Rp of 1.4 Wood $\mathrm{U} \times \mathrm{m}^{2}$. PLE, a well-known complication of Fontan circulation, may occur in up to $13.4 \%$ of patients within 10 years of the procedure and a mortality rate of $56 \%$ has been reported within five years of the diagnosis. ${ }^{1)}$ Through his clinical course, PLE occurred occasionally, for which diuretics, intravenous heparin, prednisolone, or intravenous carperitide were given. Because of the insufficient
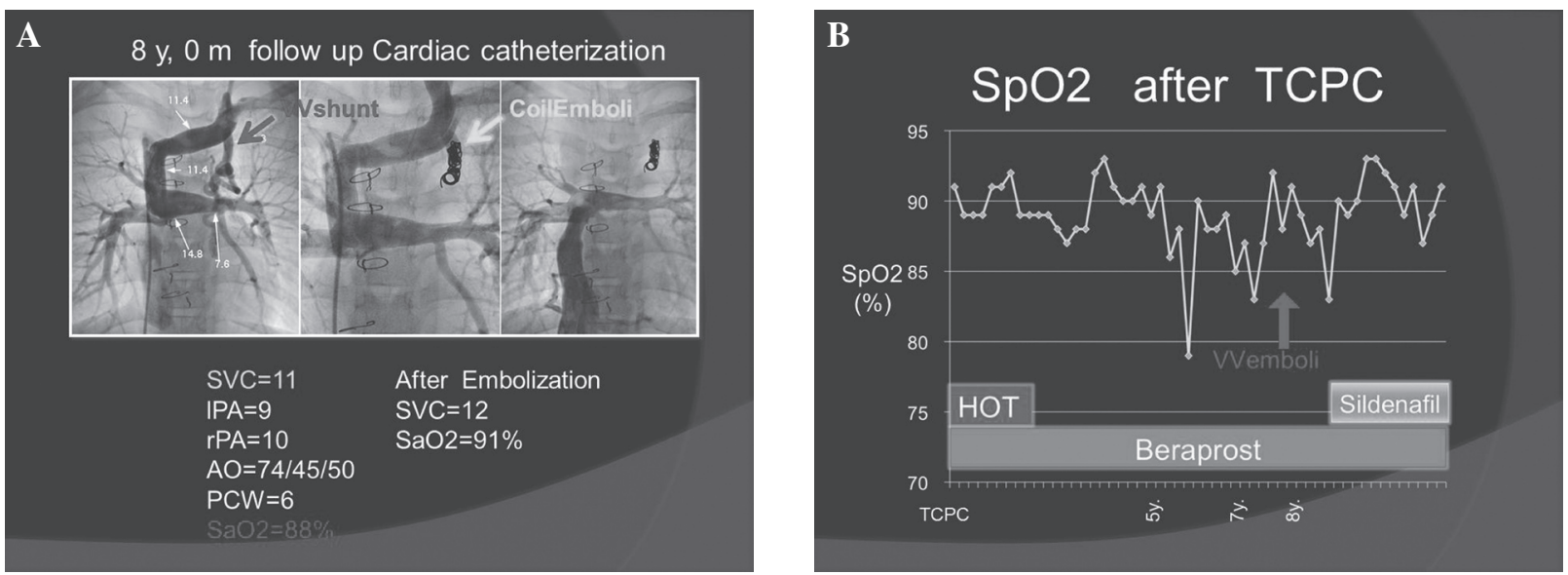

Figure 2. Angiography and catheter data of case 2 after TCPC. The oxygen saturation increased after venovenous collaterals were embolized (A). Clinical course of case 2 (B). 
A

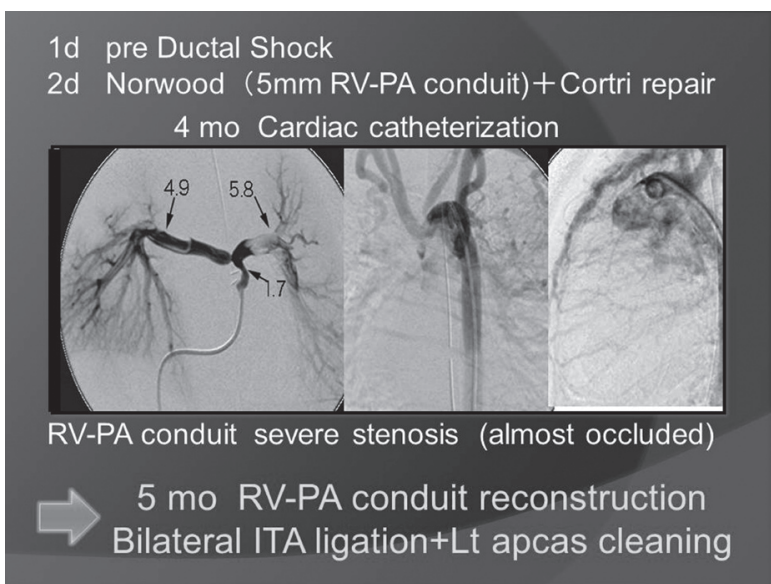

B

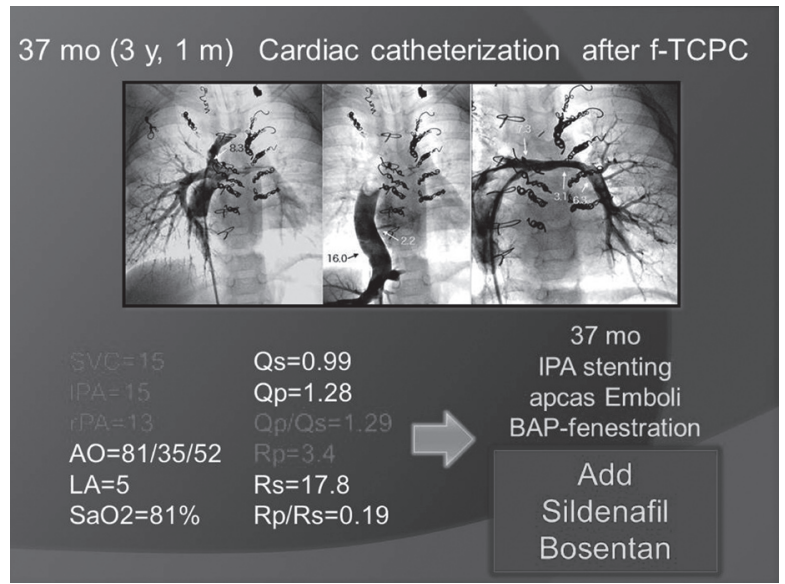

Figure 3. Clinical course of case 3 and angiography showing RV-PA conduit stenosis and massive MAPCAs (A). Angiography and catheter data of case 3 after fenestrated Fontan operation (B).

cavo-pulmonary circulation and poor control of the PLE, fenestrated TCPC was performed at the age of 30 months, and administration of beraprost was begun. At the age of 37 months, left pulmonary arterial stenting was performed, the remaining aorto-pulmonary collateral arteries were embolized, and the bending angle of the palatal cortex fenestration was increased. Sildenafil and bosentan were subsequently added (Figure 3B). At the age of 60 months, almost two years after the intensive operations and medical therapies, PLE persisted. Embolization of the aorto-pulmonary collateral arteries and fenestration over an expanded area were performed repeatedly, and the sildenafil dose was increased to $1-2 \mathrm{mg} / \mathrm{kg}$. Currently, at 70 months, PLE continues to wax and wane. For cases such as this, all procedures or medical therapies will be ineffective.

\section{DISCUSSION}

Indications and contraindications for a Fontan procedure: Fontan procedures have been applied to many variations of cardiac malformations and have helped to improve patient clinical conditions and outcomes. In Shizuoka Children's Hospital, anatomical abnormalities that disturb the final operation, such as pulmonary artery stenosis, atrioventricular valve regurgitation, and collaterals, are primarily surgically or interventionally modified. When pulmonary vasculature or pulmonary vascular resistance is the problem, high pulmonary resistance is managed using an anti-pulmonary hypertensive medical therapy. This anti-pulmonary hypertensive therapy extends the indications for Fontan procedure.

Fontan procedure issues for Down syndrome patients: Very little information is available to guide the management of a patient with trisomy 21 after Fontan operation. ${ }^{2)}$ However, patients with Down syndrome may have laryngomalacia, gas exchange problems, airway obstructions, and gastroesophageal reflux, which make surgery difficult ${ }^{3)}$ and are associated with higher mortality. ${ }^{4)}$ Therefore, every indicator must be assessed as carefully as possible.

Appropriateness of medical therapy for managing pulmonary hypertension: For candidates for the Fontan procedure as well as patients with established Fontan circulation, maintaining the pulmonary vascular bed in good condition and sufficiently suppressing pulmonary arterial pressure are very important. In recent years, various options for controlling pulmonary arterial hypertension have been reported. ${ }^{5,6}$ A large-scale, randomized prospective clinical study with and without agents for pulmonary arterial hypertension may provide truly useful information. To achieve this goal, clinical studies with sophisticated protocols are needed.

\section{ACKNOWLEDGMENTS}

The authors would like to thank Springer Healthcare and Dr Jane Charbonneau for providing English-language editing, which was funded by Actelion Pharmaceuticals Japan Ltd.

\section{REFERENCES}

1. Feldt RH, Driscoll DJ, Offord KP, et al. Protein-losing enteropathy after the Fontan operation. J Thorac Cardiovasc Surg 1996; 112: 672-80.

2. Gentles TL, Mayer JE, Gauvreau K, et al. Fontan operation in five hundred consecutive patients: factors influencing early and late outcome. J Thorac Cardiovasc Surg 1997; 114: 376-91.

3. Wada N, Takahashi Y, Ando M, Park IS, Sasaki T. Single ventricle repair in children with Down's syndrome. Gen Thorac Cardiovasc Surg 2008; 56: 104-8.

4. Gupta-Malhotra M, Larson VE, Rosengart RM, Guo H, Moller $\mathrm{JH}$. Mortality after total cavopulmonary connection in children with the down syndrome. Am J Cardiol 2010; 105: 865-8.

5. Goldberg DJ, French B, McBride MG, et al. Impact of oral sildenafil on exercise performance in children and young adults after the fontan operation: a randomized, double-blind, placebo-controlled, crossover trial. Circulation 2011; 123: 1185-93.

6. Ovaert C, Thijs D, Dewolf D, et al. The effect of bosentan in patients with a failing Fontan circulation. Cardiol Young 2009; 19: 331-9. 